\title{
Research on Advanced Oral Chinese Curriculum From the Perspective of Constructivism Theory*
}

\author{
Dan Zhou \\ Guizhou Light Industry Technical College, Guiyang, Guizhou, China; \\ Adamson University, Manila, Philippines \\ Yao Li \\ Guizhou Light Industry Technical College, Guiyang, Guizhou, China \\ Xichen Nie \\ Guizhou Light Industry Technical College, Guiyang, Guizhou, China
}

\begin{abstract}
With the rapid development of globalization, the advanced oral Chinese curriculum becomes more and more important in international education for the foreign students of the senior class in China. Aiming to address the problems in the teaching of oral Chinese curriculum, this paper uses the literature method, comparative analysis method, and survey method to study the advanced oral Chinese teaching mode under the constructivism theory. With the guidance of constructivism theory, this paper concludes and provides three teaching modes including scaffolding mode, interactive mode, and task-based mode by analyzing oral Chinese teaching cases. The construction of the three teaching modes can do more effective functions on the advanced oral Chinese curriculum for the international education teachers to use.
\end{abstract}

Index Terms - oral Chinese curriculum, the constructivism theory, scaffolding mode, interactive mode, task-based mode

\section{INTRODUCTION}

Oral ability is a crucial ability that second language learners need to master, which is a bridge between language learning in the class and practical application. It shows a strong practical significance to the language ability of students. Oral Chinese teaching in the international Chinese language teaching is indispensable and significant. While there is a big gap between ideal oral Chinese teaching and practical teaching. More interaction is needed in teaching practice by providing proper teaching modes. In the contemporary teaching of oral Chinese curriculum, some foreign students in senior levels cannot finish their oral tasks in the oral Chinese curriculum, and even they cannot understand the teaching content, resulting in the lack of vividness and effectiveness of advanced oral Chinese. The teacher-led teaching is not conducive to the development of language skills, as language skills are not imparted by the teacher to the students, but are co-constructed by the teacher and students in an interactive process. It is important to note that speaking ability is not acquired through simple interaction. It is worth exploring ways of teaching speaking that will motivate and interest students in the acquisition of speaking ability. We need to seek solutions to change the situation, improve the effectiveness of advanced oral Chinese curriculum and ensure that the teaching of advanced oral Chinese curriculum is carried out scientifically and effectively. It is a proposition that should be reflected upon in today's teaching reform process. To improve the quality of the oral Chinese teaching curriculum, this paper chooses the constructivism theory as a theoretical foundation and explores effective teaching modes of oral Chinese curriculum. As such, by analyzing the data collected from foreign students, this paper seeks effective teaching modes to improve the teaching of advanced oral Chinese curriculum aiming to address the problems in the teaching of advanced oral Chinese. It applies comprehensive research methods, especially literature research method, comparative method, and survey method with research instruments of interviews, case studies, and questionnaires to study advanced oral Chinese curriculum from the perspective of constructivism theory. This paper identifies proven teaching modes through observations during the curriculum teaching design for foreign students in the senior class. Finally, three effective modes for teaching advanced oral Chinese curriculum are analyzed and derived from teaching cases. They are scaffolding teaching mode, interactive teaching mode, and task-based teaching mode.

\section{LITERATURE REVIEW}

\footnotetext{
* This research was supported by the Project of National and Regional Research of Humanity and Social Science in Colleges and Universities of Guizhou Provincial Education Department "Research on Sustainable Development Countermeasures of 'Zhi \& Xing Guizhou' Silk Road Youth Exchange Programme”. (Project No.: 2022GByb002)
} 


\section{A. Teaching Study under the Constructivism Theory}

There are various studies under the constructivism theory, and most of these are in the field of oral English studies. According to Huang (2007), constructivism appeared in the fields of philosophy and psychology at the very beginning, and now it has been widely applied in the educational circle. Researchers believe that one of the most important developments in educational research is that constructivism theory plays an increasingly important role in teaching and learning. Constructivism learning overcomes the superficial, isolated, and mechanical learning of knowledge different from traditional learning. It emphasizes the active, social, and contextual nature of teaching and learning, and is consistent with our current educational concept of cultivating creative, autonomous, and cooperative talents. Huang's study took the ongoing college English teaching reform, and also adopted the previous researches as a reference. It aimed to improve the teaching effect of grammar and helped students to develop their language ability and oral ability simultaneously. Based on the constructivism theory, here the study told us six principles of college English grammar teaching. Gijbels et al. (2006) thought the students can find constructivist learning as the best way to acquire knowledge in a new environment, and they used seven key factors to do experiments, and the data was measured by the D-Index. Thus, we find that constructivist learning is an effective learning way in a new environment, and it is better than the ways in the conventional learning environment. Yuhaniz et al. (2019) used a pilot experiment to study the interests in history class. They used board games in the history teaching curriculum so that the history learner can develop collaboration, promote engagement in class, and create critical thinking processes under the constructivist teaching environment. They used observations, questionnaires, and in-depth interviews to conclude that the students are more active when using the constructivist way to teach in the classroom. From the studies above, we can easily find a lot of teaching studies that are based on the constructivism theory to lead the students to have more interest in learning nowadays. As such, it is necessary to take the constructivism theory into the oral Chinese curriculum to develop an effective approach. In other words, the constructivism theory is a valuable theory that we can apply in teaching practice.

\section{B. Constructivist Teaching Study in Chinese Education}

Liu (2018) used constructivism theory to study the tea culture curriculum design of Chinese learning. In the same year, Yan studied the application of "digital Story" in Chinese Culture courses for international students, and she also used constructivism theory. Gao (2011) put forward the cultivating strategies of automatic learning ability for central Asian students from the perspective of teachers. All of these materials can provide a deep research foundation. Huang (2014) wrote the article called New research progress of intercultural adaptation of international students in recent ten years, which reviewed the collection and translation of foreign literature on cross-cultural psychological adaptation since 2000 and sorted out the literature from the aspects of research objects, research methods, influence factors, adaption process, methods, and measures. Cross-cultural factors included cultural reasons and individual reasons, and they are dynamic and repeated adaptation processes. Here the specific measures are put forward for education in China. Under the constructivism theory, Liu (2021) conducted a teaching experiment for a semester of 92 students in Grade four of primary school to investigate their learning attitude, learning motivation, and teachers' professional quality. Gu (2021) used constructivism theory to construct the concept of teachers, students, and knowledge acquisition. The education in domestic universities is to cultivate such compound talents to meet the needs of economic growth and social development and to organize the "students' centered" teaching to cultivate more high-level compound talents with both professional knowledge and higher English level, which can respond to the new challenges posed by economic globalization and international competition better. From this perspective, we find there are more than 6000 papers about the constructivist teaching methods, especially most of which pay more attention to the English curriculum teaching, English curriculum teaching modes, oral English teaching methods. For example, interactive teaching research catches more eyes in teaching studies.

\section{The Study of Oral Teaching}

Wu et al. (2007) used constructivism theory to guide intermediate oral English teaching that can lead the learners out of learning difficulties, and cultivate students' language communicative ability in a better way. With the gradual improvement of the learning interest of the Chinese language, Xiao (2014) proved the theoretical and practical significance of oral Chinese teaching under constructivism theory. Based on this background, he introduced constructivism learning theory into the teaching of oral Chinese curriculum, trying to cultivate the initiatives of oral Chinese learning. With constructivist theoretical instruction, we can easily promote the improvement of oral Chinese level and language application abilities. Yuan et al. (2021) thought that the oral communicative ability cannot meet the actual needs, and the traditional Chinese oral English teaching effect seems not ideal. Language teaching and learning must be carried out in a certain environment, just like the virtual reality technology environment in their study, and we analyze the characteristics, pros, and cons in Oral English teaching. Finally, we find new constructivism teaching methods can do more positive significance to oral teaching in the class. As well, using constructivism theory to study oral Chinese teaching is a hot spot research field. Lots of scholars studied different levels of oral Chinese learning. Wang (2018) studied the teaching design of primary oral Chinese teaching in Thailand from the perspective of the Constructivism scaffolding approach. Liu (2021) used interactive teaching mode under constructivism theory to study the network broadcast teaching of advanced oral Chinese curriculum. Sun (2012) found the dominant principle of teaching method which is "learning by doing" under constructivism theory, emphasizing the practical and communicative nature of 
language acquisition, and advocating to use the acquisition of target language in the process of completing teaching tasks. It shifted from the traditional teacher-centered single mode in a student-centered mode, inserted more communicative activities in the class, let the students participate independently and collaborate interactively, and realized the language learning goal. In the study of oral teaching scope, we study from two dimensions under the constructivist background. One is the oral English teaching; while the other is the oral Chinese teaching. Facing the current situation of oral Chinese teaching, we gathered materials at least three levels. The first level is elementary oral Chinese. The second level is intermediate oral Chinese. And the third one is advanced oral Chinese learning. The close connections between oral Chinese learning and constructivism theory are found.

The above research practices based on constructivism theory, and different research methods have been used to explore different types of teaching and speaking instruction. It is found that constructivism theory is widely used. More importantly, it can be of great importance and value in guiding the construction of students' language skills in practical teaching, especially in oral language teaching. The Constructivism theory emphasizes that language skills are not imparted by teachers, but are actively acquired by students in certain contexts with the help of teachers or peers. In the construction of language skills that are both interactive and contextual, the embedding of oral tasks has a facilitating effect on the improvement of Chinese speaking ability. However, existing research is inadequate in analyzing how constructivism theory can be applied to practical cases of advanced oral Chinese curriculum. Therefore, based on the constructivism theory, this paper aims to explore the practical value of teaching modes and methods based on actual cases in the advanced oral Chinese curriculum.

\section{METHODOLOGY}

\section{A. Literature Research Method}

Based on the search of plenty of Chinese and foreign research literature mainly including articles, classical books, magazines, and research results, etc., this paper takes a comprehensive discussion on the teaching modes of advanced oral Chinese teaching from the perspective of constructivism theory and has a deep analysis of the teaching situation to the advanced oral Chinese curriculum. Through the CNKI platform, we get lots of excellent graduate papers for master's or doctor's degrees, and also search materials from Springer, Elsevier to get abundant articles. A large number of previous research materials and views that the authors collected laid a solid research foundation for this paper.

\section{B. Comparative Analysis Method}

The paper gathers data from a senior international class of 10 students about their studying goals, learning interest, and learning effect. It uses interviews and team discussion ways to know more about the different teaching effect in oral Chinese learning. Finally, we seek effective teaching modes about how to improve learning interests in advanced oral Chinese teaching and convert the conventional teaching mode from teacher-centered to student-centered mode, which can stimulate the international students to learn oral Chinese more effectively. In this part, we get comparative analysis among different students when facing a certain oral Chinese curriculum design, which is one comparative dimension; while the other comparative aspect is to compare the oral Chinese learning with oral English learning, and there are similarities of teaching skills and indeed we can take each other as a reference.

\section{Survey Method}

The survey method includes interviews, case studies, questionnaires. This paper will choose several students to interview and to observe their emotions and learning goals in their oral Chinese teaching class. For different students, they will show different attitudes towards the same teaching design. In this way, we can improve the teaching case in a more proper way and make it excellent in the oral Chinese teaching. Furthermore, questionnaires are the necessary way in a research study, the paper will design several questionnaires to survey about the research topic and conclude some effective teaching modes to give the teachers of the senior international students class to use. Based on the understanding of the students and the steps of teaching, we design the questionnaires containing the basic situation of the study subjects, their understanding degree of the teacher, and the using process of the practical teaching.

\section{TEACHING MODES}

\section{A. Scaffolding Teaching Mode}

Vygotsky (1978) emphasizes interaction and engagement with the target language in a social context based on the concept of Zone of Proximal Development (ZPD) and scaffolding. In other words, learning is best achieved through the dynamic interaction between teacher and the learner and between learners. With the teacher's scaffolding through questions and explanations, or with more capable peers' support, the learner can move to a higher level of understanding and extend his or her skills and knowledge to the fullest potential. Therefore, scaffolding teaching mode as a new teaching method of constructivism theory has a great advantage, especially for oral language teaching. The core concept of scaffolding is an activity guided by the teacher. It provides enough time and chance to let the students learn by themselves, which can be defined as student-centered learning. In the oral Chinese curriculum, the teacher designs scaffolds according to the teaching context and content rightly to guide the students to improve in accordance with the 
knowledge framework and to achieve the improvement of language skill. In the oral Chinese curriculum, this scaffolding teaching mode can attract students to open their mouths to speak and make clear their learning goals. The scaffolding teaching mode has strong interactivity, and it will show a reciprocal interaction when the scaffolding is fixed properly.

The process of using the scaffolding teaching method to teach is the process of selecting, building, and removing the scaffolds based the designed context. In the teaching practice, teachers can think about what the teaching contents will be involved, then design scaffolds to support and guide the students to develop themselves. The operation process of the scaffolding teaching method is generally composed of six components. They are selecting a theme, building scaffolds, entering a situation, independent exploration, cooperative learning, and effects evaluation. Under constructivism theory, the introduction of theoretical scaffolding teaching method into advanced Oral Chinese teaching is helpful to form a more practical oral Chinese teaching method. This paper uses survey and interview methods to verify the scaffolding teaching mode. Given advanced oral Chinese teaching in the scaffolding teaching mode, the paper analyzes the general information of learners, the influencing factors, and relevant factors of application effect, all of which will provide a beneficial reference for advanced Oral Chinese teachers.

Taking the case Lesson 8 Beautiful Heart, Everybody Has from the advanced oral Chinese curriculum in Developing Chinese (The Second Version) in the advanced oral Chinese teaching for example, target students are assigned to make a speech by themselves, but they do not have enough language knowledge including sentence patterns, phrases, words or connective words, then scaffolding mode here is a good way to take as follows:

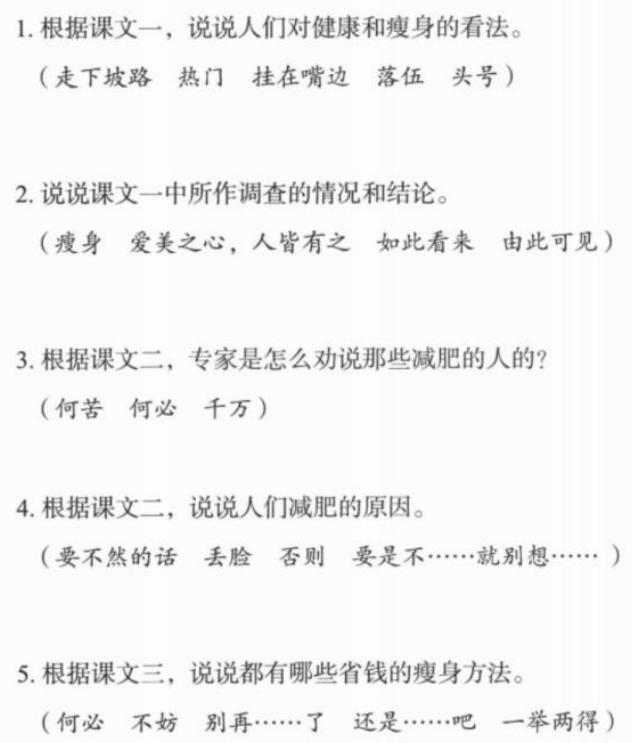

Figure 1. Some Scaffolding Phrases or Words for the Task

In this task, it may be difficult for students to find proper sentence patterns, phrases, words, or connective words to construct their own language in this lesson, so these words or phrases shown in Fig. 1 are chosen as the scaffolds, such as the sentence patterns like "If not do like this" and "but for", and phrases like "lose face" and "or else" in this oral Chinese curriculum. As scaffolds for the reason of excise, they are classified into sentence patterns, phrases, words and connective words shown in Fig. 2. All of these scaffolds in this case can consist main meaning of the passage.

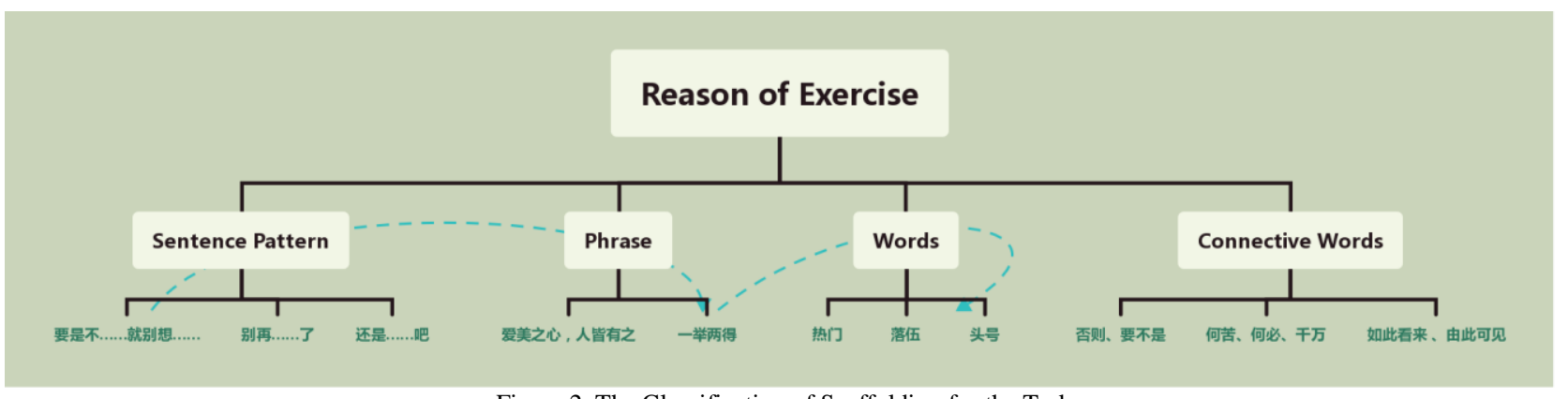

Figure 2. The Classification of Scaffolding for the Task

With the scaffolds provided in the teaching case, the students may have confidence and interest to finish this task, which can drive them to tell what the passage is about and express the main ideas in the passage. So the scaffolding mode is a good way for the students to construct their own language of what they want to express. Then the students can use these scaffolds to accomplish their five-minute speech in a clear-thinking method. According to the six components of 
the scaffolding approach, the theme selection and scaffolding building components of this teaching case have been completed. And the context entry and independent exploration components are the processes of self-construction of students' language skills supported by the scaffolding. After the self-construction, students engage in cooperative learning based on the language foundation they have already constructed. The subsequent evaluation of the results is the processes of language output, i.e. the formation of advanced oral Chinese ability. The Cooperative learning is the processes of consolidating self-constructed language skills through peer interaction, in which peers provide each other with scaffolding to make them take the initiative to cooperate and communicate. The evaluation is a process of teacher-student and student-student interaction with the scaffolding of the output of the teacher or peers. Here we can easily get the conclusion that the scaffolding mode is an effective teaching method for the advanced oral Chinese teacher to organize their curriculum teaching.

\section{B. Interactive Teaching Mode}

A language is a communicative tool in real interaction. Through investigation and interview, it is of great significance for teachers to apply interactive teaching mode to carry out advanced oral Chinese teaching. The interactive teaching mode emphasizes students' dominant position and advocates teacher-student interaction, students-student interaction and other multidimensional interactions. It can achieve the interactive teaching behavior in the oral Chinese class, stimulate students' participation enthusiasm, and improve the interactive effect of oral Chinese teaching. In the design of interactive activities, we should pay attention to the characteristics of multidimensional interactions, improve the students' oral communicative ability, and achieve the teaching goal of the curriculum. Ur (1996) describes ten forms of interactive classroom activities including group work, whole-class interaction, and self-assessment. In other words, there are multidimensional forms of classroom interaction that teachers can flexibly choose to use in their teaching practice. In addition to the traditional teacher-student question-and-answer format, peer interaction may be a more effective form of interaction to facilitate students' meaning construction. Fan and $\mathrm{Xu}$ (2021) encourage teachers to give students freedom to choose familiar peer partners in classroom group activities to enhance student' emotional experience, negotiate meaning, and strengthen peer support and social connections. Nowadays, the researchers realize that interactive teaching has become a modern teaching reform method under the constructivism theory, and will inevitably become a new teaching mode suitable for oral teaching.

A majority of the oral English curriculum for the international students in the new era turn to online courses but seriously lack interaction. Therefore, the basic principles of interactive teaching and interactive types of specific teaching processes are used to explore the teaching modes. The constructivism theory is used to study how online interactive teaching is implemented, how the online interactive process occurs, and what the specific contents in the interactive process are. It can provide a reference and improve the multidimensional interaction of the advanced oral English class in the current practical teaching. Also, it is an effective oral Chinese teaching method currently. We need to change the teacher-centered teaching mode to a student-centered teaching mode. Comparing these two teaching modes we can easily find the advantages of the interactive teaching mode guided by the construtivism theory.

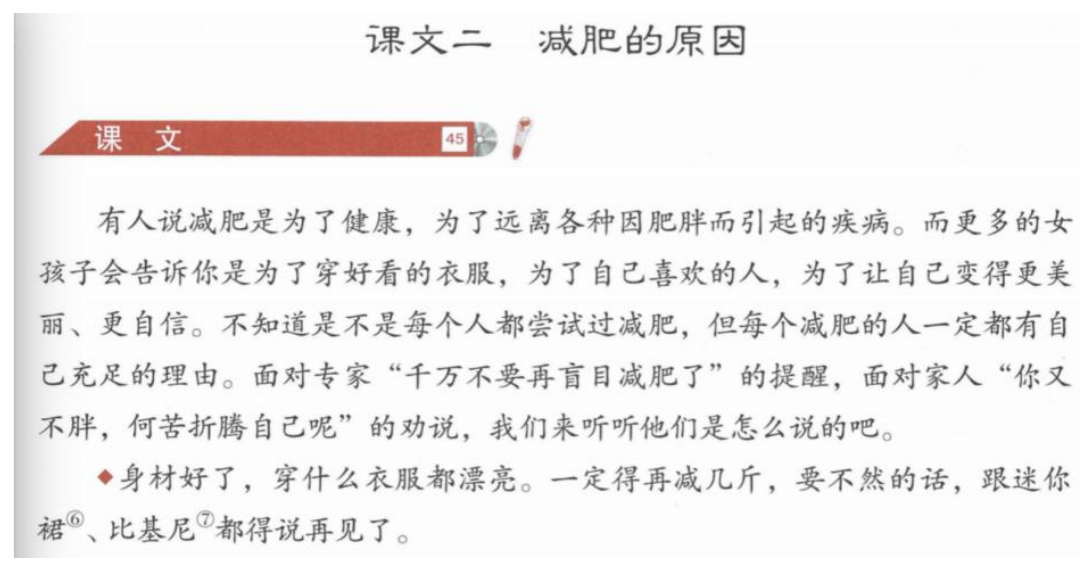

Figure 3. The Extract of Teaching Text

Taking the teaching case in the second passage of Lesson 8 in the Developing Chinese(The Second Version) shown in Fig. 3 for example when teaching in the class, the teacher firstly opens the camera online and says hello to the students and ask students to read sentences one by one according to the topic. And he starts to interact with students.

Teacher: Why do people want to lose weight nowadays?

Students: Keep healthy, avoid disease, get a handsome boy friend or beautiful girl friend etc.

Teacher: Yes, you are right! All of these reasons need people want to lose weight in order to pursue health and beauty.

(The teacher shows some pictures, i.e. some elegant women, some superstar, and so on)

Apart from simple question-and-answer interaction between teachers and students, peer or student-student interaction is also an essential mode of teaching interaction in the advanced oral Chinese curriculum. In this teaching case, the teacher can also design a peer-to-peer interview activity about weight loss for students to express their own views while 
strengthening student-student interaction, truly achieving the communicative function of the language. At the same time, the students can experience the fun and meaning of using Chinese to communicate.

This interactive teaching mode can make the students interested in learning passage and they will really know more about cultures of the society from the oral Chinese curriculum teaching. Therefore, we can teach oral Chinese in the interactive teaching mode, which can fully activate students' learning vitality. It is worth noting that the creation of any interactive modes needs to be designed and implemented by language teaching practitioners according to the teaching objectives, teaching activities and teaching plans. Also, it involves the analysis and processing of the teacher's teaching content texts as well as the retrieval, reading and collection of language materials, etc. At the same time, the flexible use of modern information technology can add interest to the creation of the interactive teaching mode.

\section{Task-based Teaching Mode}

The theoretical essence of constructivism theory is that learners use previous knowledge and experience to acquire new knowledge and experience, then the newly acquired knowledge and experience can deepen the learners' understanding of the previous knowledge in reverse. Through repeated interactive task-based teaching, knowledge can be constructed well under the constructivism theory. The main idea of the task-based teaching mode is that the teacher can design the real task by giving the reasons shown in Fig. 4 according to the students' learning needs. In this way, it can activate learners' existing knowledge and cognitive structure, and stimulate their desires of acquiring new knowledge and getting information. At last, learners can finish the tasks successfully, which means they have already completed the construction of the new knowledge.

The task-based teaching is embedded in almost every aspect of teaching and learning, and there are numerous studies on the application of task-based teaching. In other words, as long as there is teaching, tasks exist. However, how to effectively use the task-based teaching mode in the teaching of advanced oral Chinese is a question worth exploring. The task-based teaching mode is an important teaching method under the constructivism theory. It concretely embodies in the communicative teaching method and the theory of knowledge acquisition. We can use the task-based teaching method to make the students acquire more specific knowledge and finish ability training in the senior oral Chinese teaching curriculum. This kind of teaching method can lead the students to construct more knowledge and promote students' speaking ability.

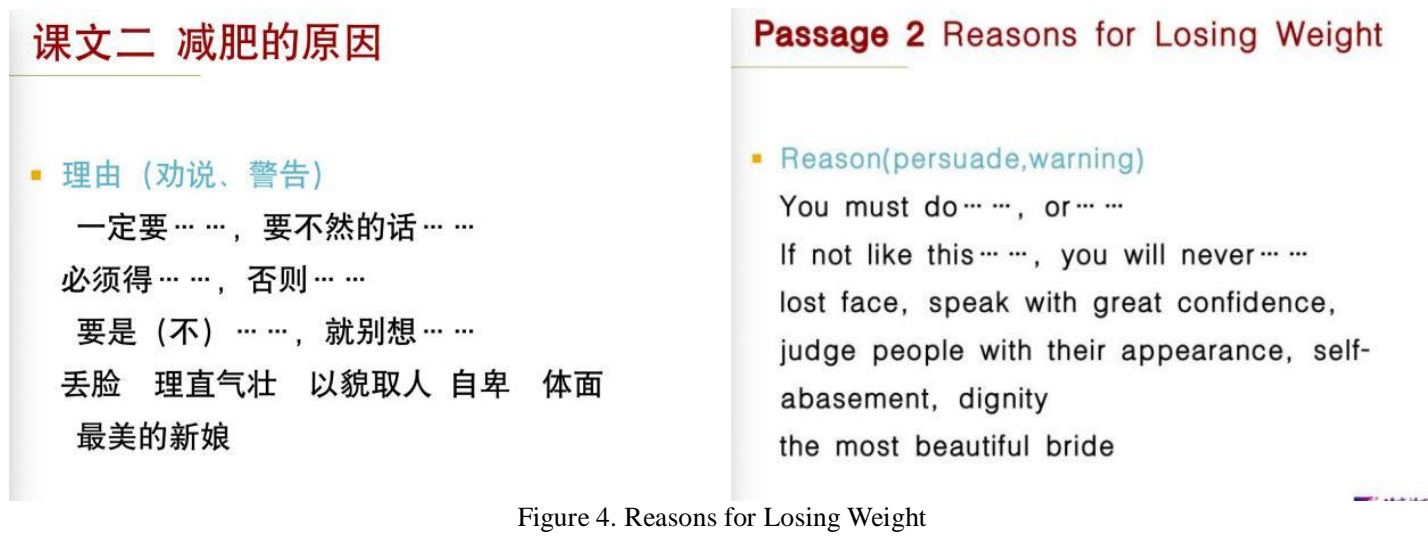

In the second passage of Lesson 8 Reason for Losing Weight in the Developing Chinese(The Second Version), the teacher assigns the students to give a five-minute speech after reading the passage according to the students' learning needs in the advanced learning stage. We design the oral Chinese curriculum according to the task and give some keywords and the backbone sentence patterns for the international students to fill some content to form a fluent speech. We firstly make clear about the task for the students, then give them a certain time to organize dialogue by themselves. In this teaching activity, the task itself is the process of forming students' advanced oral Chinese skills. Different parts of the activity correspond to different tasks. In these activities, students are supported by scaffolding to refine each task part, ultimately forming the construction of language skills in a speaking proficiency-oriented task. It is easy to find that the task-based teaching mode is an effective way of guiding students to acquire Chinese speaking skills in a structured way.

\section{CONCLUSION}

The three teaching modes for the advanced oral Chinese curriculum are conducted by analyzing the teaching examples combined with the constructivism theory. As we can find, the constructivism theory is extremely important to language teaching. Based on the constructivism theory, this paper concludes the several teaching modes for advanced oral Chinese teaching in China. Using this theory and its specific teaching methods in practice can be of positive significance to the international oral Chinese teaching. And it can also improve the curriculum teaching level. The scaffolding, interaction, and tasks are truly indispensable components for the advanced oral Chinese curriculum. However, it should be noted that 
the three teaching modes are not implemented in an isolated way and they need to be carried out comprehensively for better teaching effect. These teaching modes under the guidance of constructivism theory can be more acceptable for students. They are beneficial to improve the teaching efficiency, and improve students' advanced oral Chinese level and communicative skills. While it may be limited by the number of samples, as more studies to explore effective teaching methods are invited to present for our international education teachers.

\section{REFERENCES}

[1] Fan, Y. M., \& Xu, J. F. (2021). Tong ban shu xi du dui tong ban hu dong zhong xue xi zhe tou ru de ying xiang yan jiu [The Study on the Impact of peer familiarity on learner engagement in peer interactions]. Foreign Languages and Their Teaching, 02,82-91+149-150.

[2] Gijbels, D., \& van de Watering, G., Dochy. (2006). New Learning Environments and Constructivism: The Students' Perspective. Instr Sci, 34, 213-226.

[3] Gu, Y. (2020). Application of Scaffolding Teaching Method in Elementary Chinese Writing Teaching [Unpublished master's thesis]. Beijing Foreign Studies University.

[4] Huang, H. (2007). Research on College English Grammar Teaching from the Perspective of Constructivism [Unpublished doctoral dissertation]. Shanghai International Studies University.

[5] Huang, Z. (2014). Research Progress of Intercultural Adaptation of International students in Recent Ten Years. Comparative education research, 36(08), 88-92.

[6] Liu, D. (2021). Research on Network broadcast teaching of advanced Oral English Class based on Interactive Teaching Mode [Unpublished master's thesis]. Yunnan Normal University.

[7] Liu, M. Y. (2018). Teaching Design of Tea Culture Course for Overseas Study in China [Unpublished master's thesis]. Tianjin University.

[8] Sun, X. Y. (2012). Exploration and Practice of Task-based Elementary oral Chinese Teaching [Unpublished master's thesis]. Guangxi University.

[9] Ur, P. (1996). A Course in Language Teaching: Practice and Theory. Foreign Language Teaching and Research Press.

[10] Vygotsky, L. (1978). Mind in Society: The development of higher psychological process. Harvard University Press.

[11] Wang, W. (2018). Research on the Design of Primary Oral Chinese Teaching in Thailand from the perspective of Constructivism Theory scaffolding Teaching method [Unpublished master's thesis]. Inner Mongolia Normal University.

[12] Wang, Y. X. (2021). Research on the Application of Scaffolding Teaching Method in Primary Spoken Chinese Classroom [Unpublished master's thesis]. Shenyang Normal University.

[13] Wu, R. (2007). Research on Intermediate oral Chinese Teaching from the Perspective of Constructivism [Unpublished master's thesis].Shanghai Normal University.

[14] Xiao, Y. S. (2014). Research on the Application of Constructivism Learning Theory to the Initiative Training of International Students' Oral English Learning [Unpublished master's thesis]. Liaoning University.

[15] Yan, Y. H. (2018). Research on the Application of "Digital Story" in The Chinese Culture Course of International Students [Unpublished master's thesis]. Shandong Normal University.

[16] Yuan X., \& Li D. (2021). The Application of Virtual Reality Technology in College English ESP Oral Teaching. In Abawajy J., Xu Z., Atiquzzaman M., Zhang X. (eds), Lecture Notes on Data Engineering and Communications Technologies. Springer.

[17] Yuhaniz M., \& Samsuddin I. (2019). Improving Student Interest Through Games in Architecture History Using Constructivism. In Mat Noor A., Mohd Zakuan Z., \& Muhamad Noor S. (eds), Proceedings of the Second International Conference on the Future of ASEAN (ICoFA) 2017 - Volume 1. Springer.

Dan Zhou, female, born on July in 1986, from Ma Anshan City, Anhui Province, China, is the researcher of this research. Zhou has earned her master's degree. She is currently an associate professor in the Guizhou Light Industry Technical College, Guiyang City, China. Zhou's academic research area is about internationalization of higher education and vocational education.

Yao Li, female, born on October in 1991, from Anqing City, Anhui Province, China, is the co-researcher of this research. Li has earned her master's degree of teaching Chinese to speakers of other languages. She is currently a mandarin teacher in the Gui zhou Light Industry Technical College, Guiyang City, China. Li's academic research area is about teaching Chinese as a second language.

Xichen Nie, female, born in July, 1994, Bijie City, Guizhou Province, China, is the participant co-researcher of this research. Nie has earned her master's degree in Colorado State University. She is currently a staff in the Academic office of Waikato International College of Guizhou Light Industry Technical College, Guiyang City, China. Nie's academic research area is about teaching English to speakers of other languages. 